\title{
Research Paper: Investigation of the Formant Structure of Persian Vowels in the Persian - Azari Bilingual Adults
}

\section{Farideh Kamran ${ }^{1}{ }^{\oplus}$, ${ }^{*}$ Seyyedeh Samaneh Mirahadi ${ }^{1}$, Banafsheh Mansuri ${ }^{2}$, Seyyed Abolfazl Tohidast ${ }^{3}$, Kosar Rashtbari ${ }^{4}$, Elahe} Panahgholi ${ }^{4}$, Aref Taghipur ${ }^{4}$

1. Department of Speech Therapy, School of Rehabilitation, Tehran University of Medical Sciences, Tehran, Iran.

2. Department of Speech Therapy, School of Rehabilitation Sciences, Iran University of Medical Sciences, Tehran, Iran.

3. Neuromuscular Rehabilitation Research Center, Semnan University of Medical Sciences, Semnan, Iran

3. Department of Speech Therapy, Faculty of Rehabilitation, Tabriz University of Medical Sciences, Tabriz, Iran.

Received: 24 Dec 2017 Accepted: 01 Apr 2018

Keywords:

Vowel, Formant,

Bilingual, Adult dtat On: Kamran F, Mirahadi SS, Mansuri B, Tohidast SA, Rashtbari K, Panahgholi E, et al. [Investigation of the Formant Structure of Persian Vowels in the Persian - Azari Bilingual Adults (Persian)]. Archives of Rehabilitation. 2018; 19(2):142-149. http://dx.doi.org/10.32598/rj.19.2.142

dof: $:$ http://dx.doi.org/10.32598/rj.19.2.142

\section{A B STRACT}

Objective Vowels are the center of syllables while formant structures are one of the most important acoustic characteristics of speech sounds that help in their articulatory and perceptual aspects. Formants represent the shape and size of the vocal tract. There exist trivial differences between the vocal tracts of different people due to which the formant structures of a vowel in one person are different from another person. Towards the end of the $20^{\text {th }}$ century, advances in acoustic science and production of digital tools introduced voice acoustical characteristics analysis as another parameter of sound analysis. These voice acoustical characteristics analyses lead to better assessment of the voices. Effective treatment of speech disorders depends on accurate diagnosis by the speech-language pathologist. The relationship between the first three formants is the main component of perceptual categorization by the listener. Therefore, the aim of the current study is to determine and compare Persian vowel frequency of first, second and third formants in bilingual adults.

Materials \& Methods A cross-sectional study was performed on 50 participants (25 males and 25 females) aged 18 to 24 years. Bilingual students from Tabriz University of Medical Sciences were selected by an easy and non randomized sampling method, where the average of the first, second and the third formant frequencies for each of the six Persian vowels were taken into consideration. Data collection involved a demographic questionnaire (age, gender, bilingualism, and diseases related to the speechbreathing mechanism), the consent form, a computer equipped with PRATT Software and one sound recorder set. The data were analyzed using SPSS V. 18 software. When the data were normally distributed, independent t-test was used; otherwise, Mann-Whitney test was used.

Results This study showed that the maximum and minimum values of F1 in the males and females are /æ/ and /i/ vowels. The maximum and minimum values of F2 are /i/ and /o/ vowels in both the sexes, whereas, the maximum and minimum values of $\mathrm{F} 3$ are /i/ and /æ/ vowels in males and /i/ and /a/ vowels in the females.

Conclusion According to the results of this study, / æ/ and /i/ vowels in both the sexes are the most open and closest. /i/ and /o/ vowels in both the sexes are the most front and most back vowels. The most spread vowel in males and females is /i/ but the roundest vowel is /æ/ in the males and /a/ vowel in females.

\section{* Corresponding Author:}

Seyyedeh Samaneh Mirahadi, MSc.

Address: Department of Speech Therapy, School of Rehabilitation, Tehran University of Medical Sciences, Tehran, Iran.

Tel: +98 (914) 1972869

E-Mail: s.mirahadi@yahoo.com 


\section{بررسى ساختار سازهاى واكههاى زبان فارسى در بزرّىسالان دوزبانه آذرى فارسى}

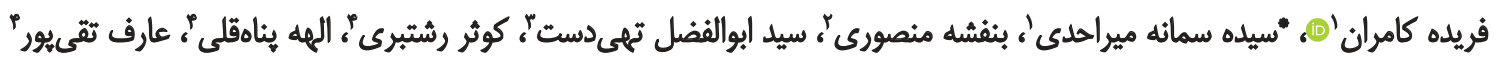

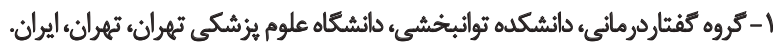

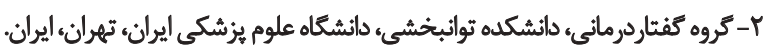

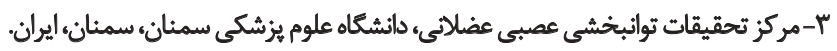

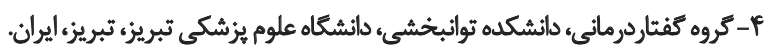

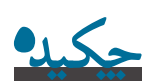

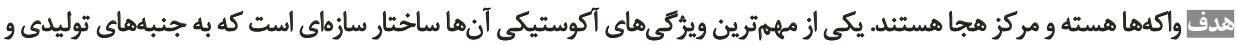

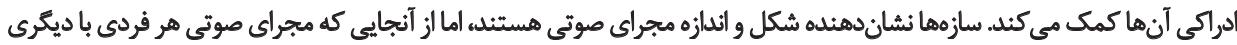

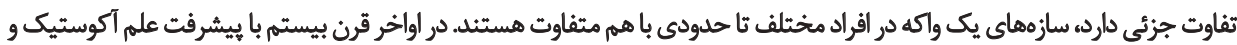

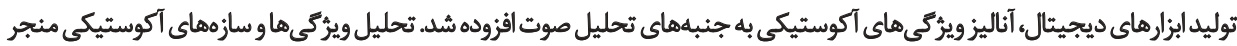

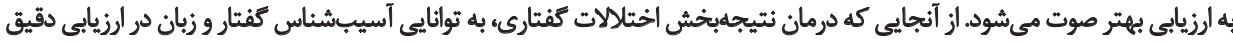

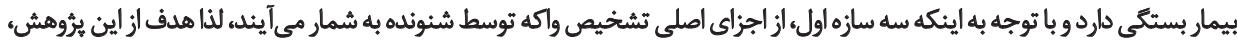

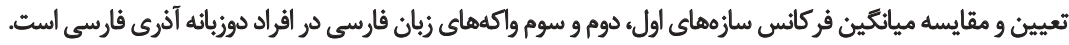

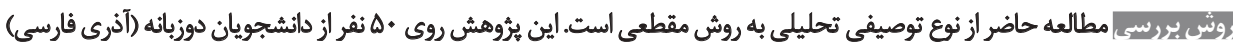

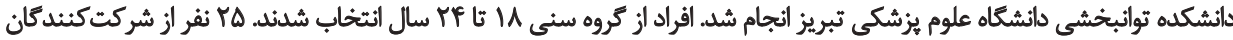

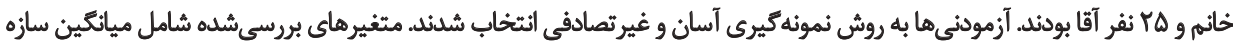

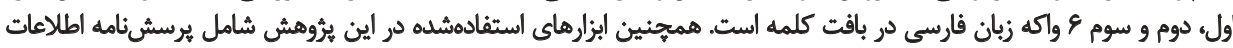

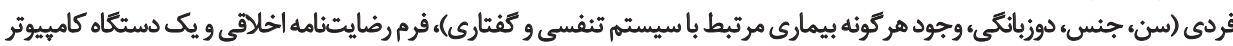

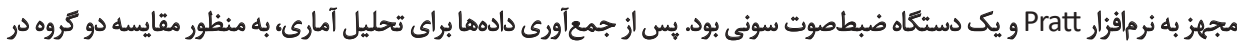

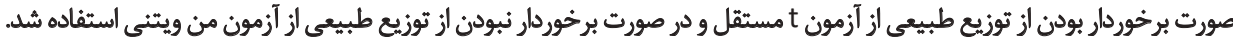

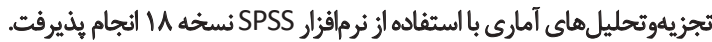

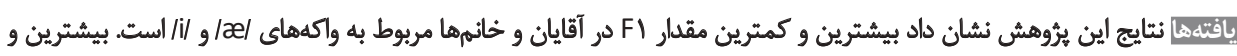

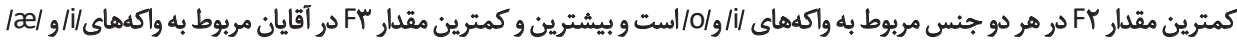

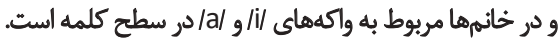

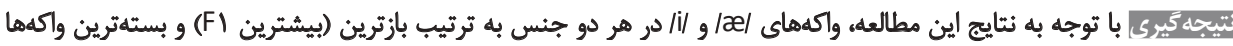

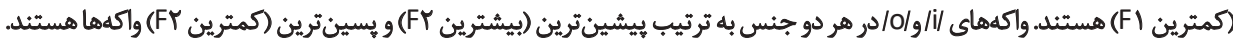

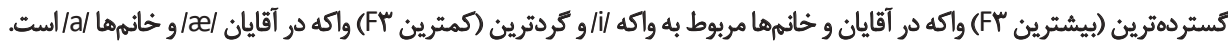

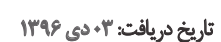

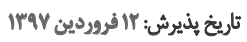

اين به توانايى كوينده براي توليد صحيح آواها بستكى دارد [T].

مقدمه

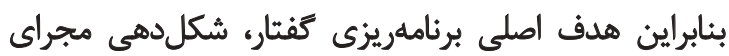

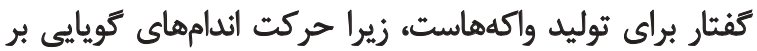

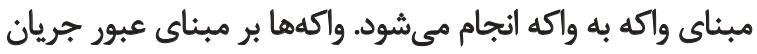

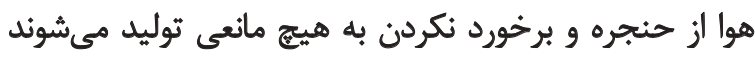

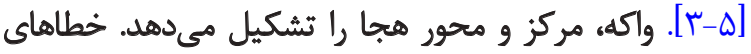

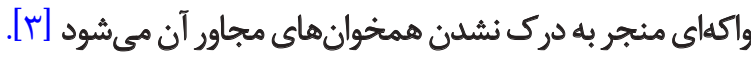

در اثر عبور جريان هوا از حنجره و ارتعاش تارهاي صوتى، صدا

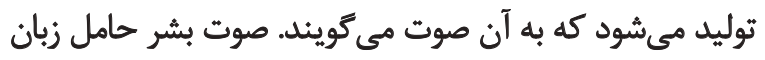

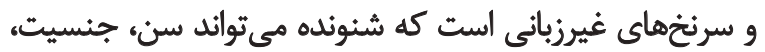

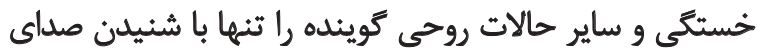

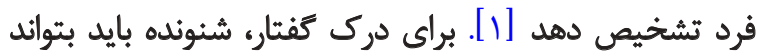

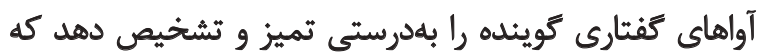

" نويسئده مسئول:

سيده سمانه ميراحدى مئول: نشانى: كروه كففتاردرمانى، دانشكده توانبخشى، دانشعاه علوم يزشكى تهران، تهران، ايران. تلفن: رايانامه: S.mirahadi@yahoo.com 
بسيارى از مطالعات ويرُّى هاى آكوستيكى واكهها را بررسي

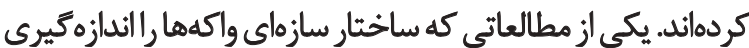

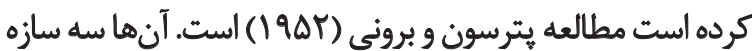

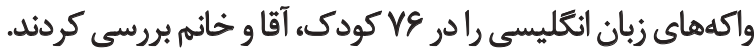

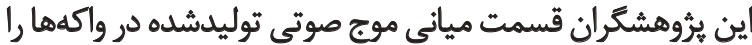

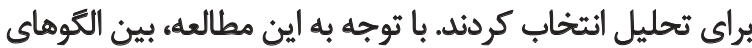

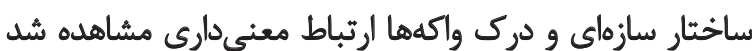

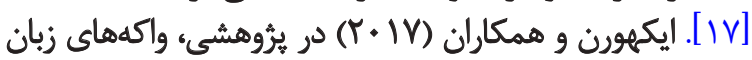

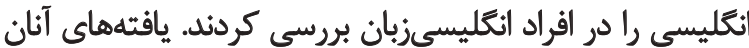

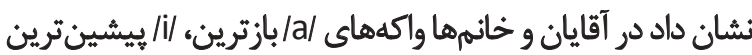

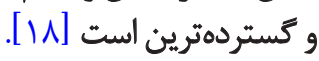

در مطالعه محمدى و همكاران (Iإ) (Y)، بيشترين و كمترين

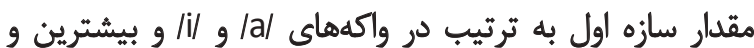

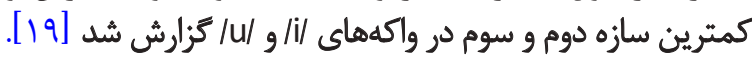

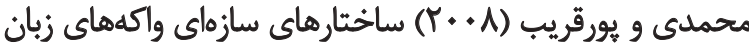

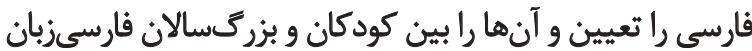

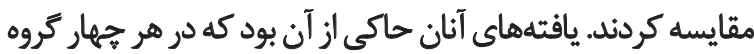

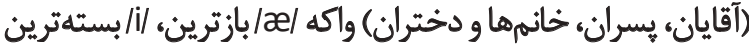

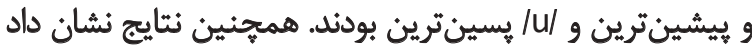

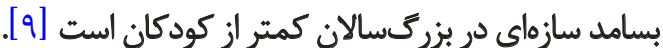

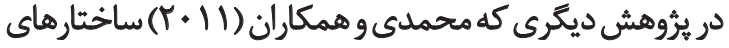

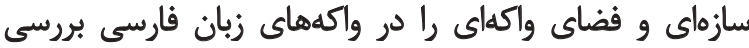

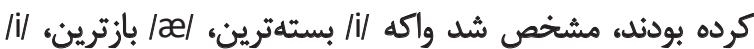

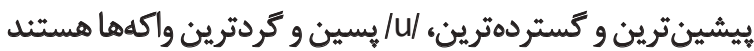

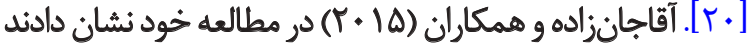

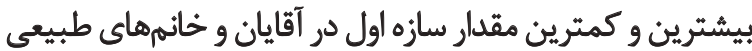

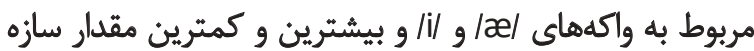

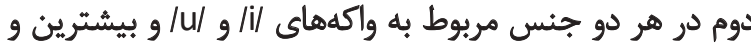

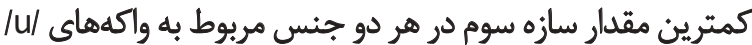

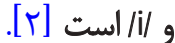

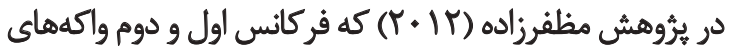

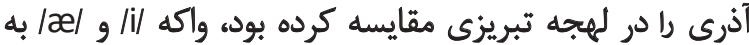

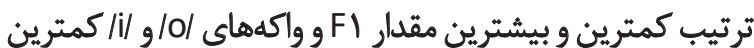

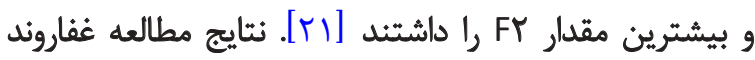

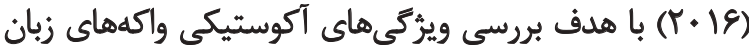

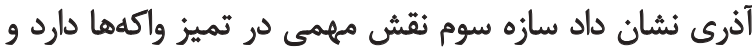

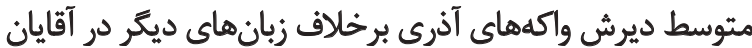

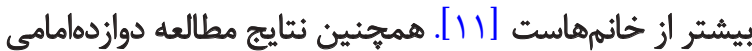
(T.IV)

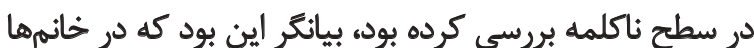

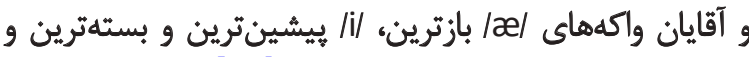

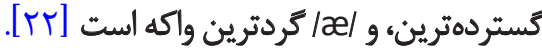

بنابراين ضرورت تحليل آواييى واكهها از نظر آواشناسى فيزيكى

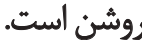

يكى از مهمترين ويرگتىهاي فيزيكى واكهها ساختار سازهاي

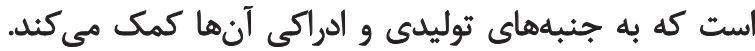

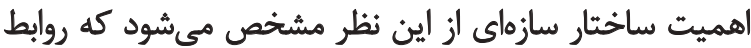

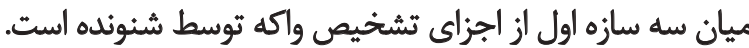

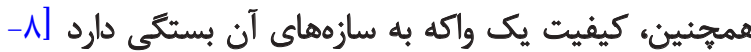

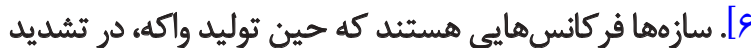

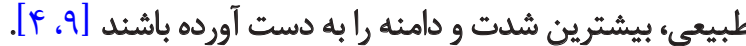
هر واكه هندين سازه دارد كه به طور كلي سه سازه اول در

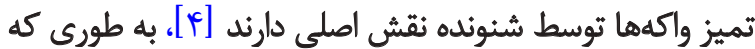

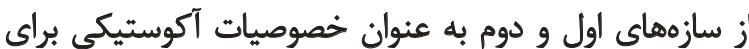

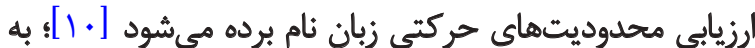

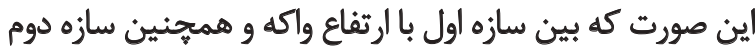

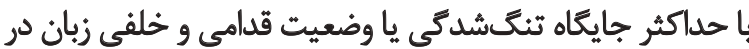

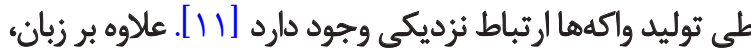

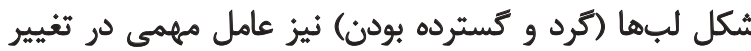

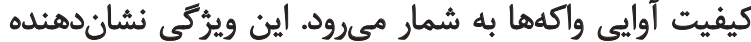

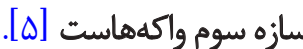
جانسون معتقد است كه اين سازهها نشاندهنده شكل و اندازه

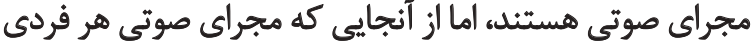

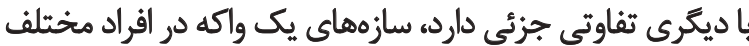

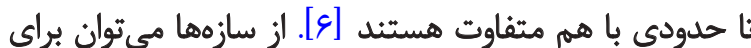

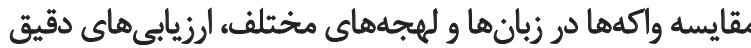

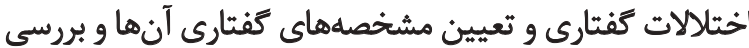

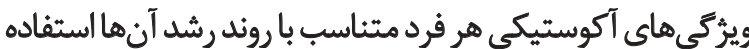

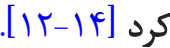

دوزبانكى يديدهاي است كه در جوامع بشر رو به كَسترش

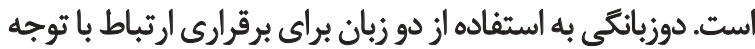

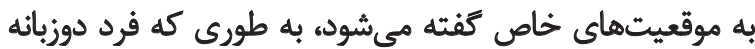

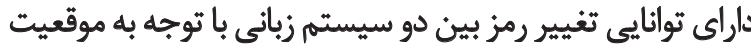

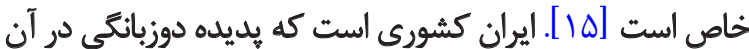

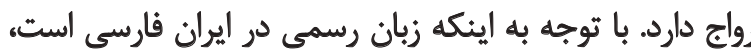

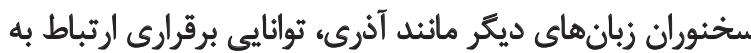

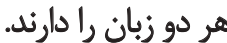

زبان آذرى شامل 9 واكه است كه تنها در 9 واكه با زبان

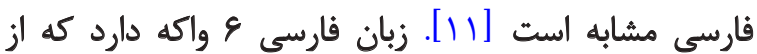

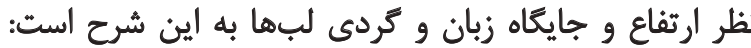

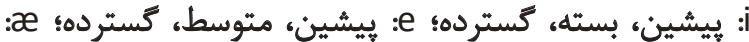

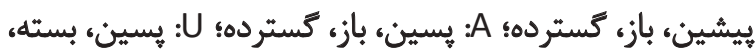

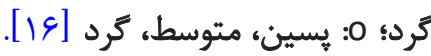


استفادهشده در اين يُؤهش شامل يرسشنامه اطلاعات فردى

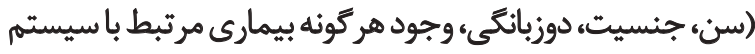

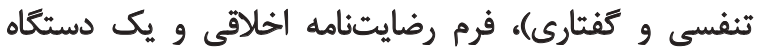

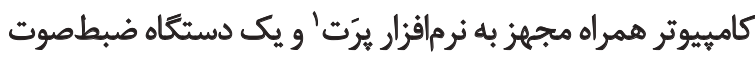

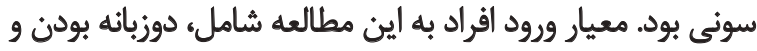

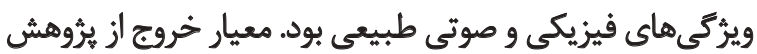

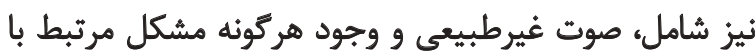

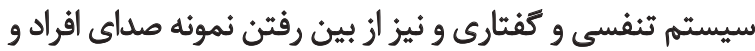

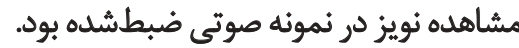

به منظور تشخيص ويرَّىهاى صوتى طبيعى، يك نمونه

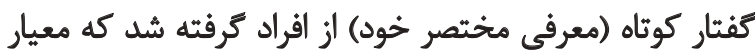

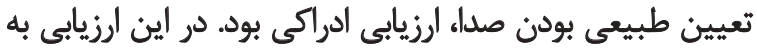

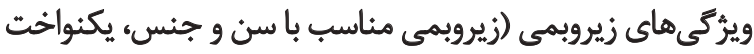

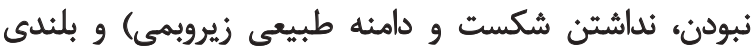

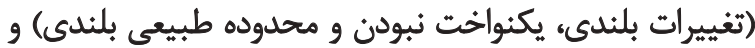

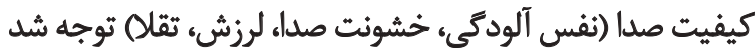

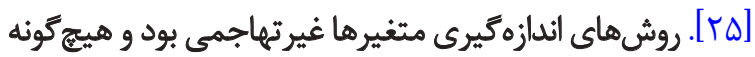

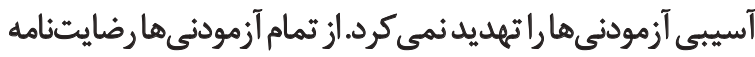

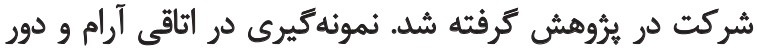

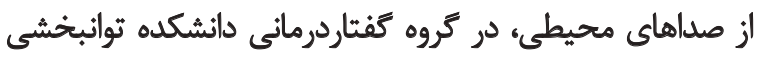

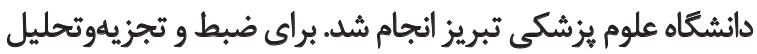

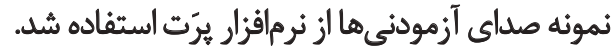

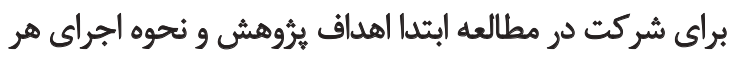

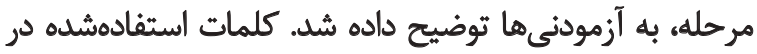

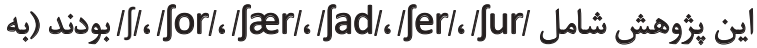

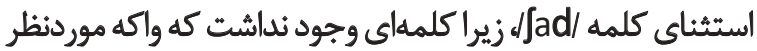

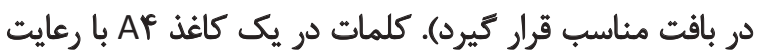

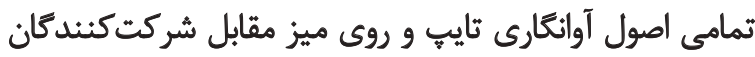

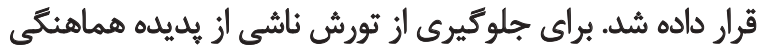

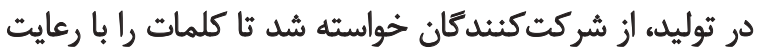

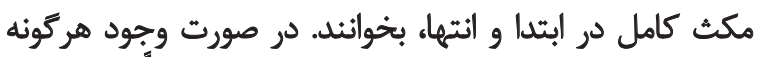

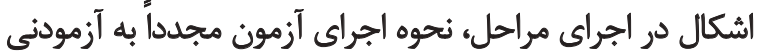
توضيح داده و اجراى آزمون تكرار مى نشيد.

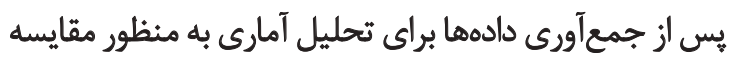

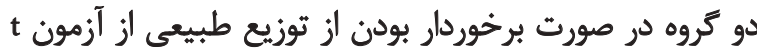

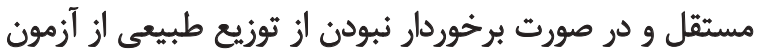

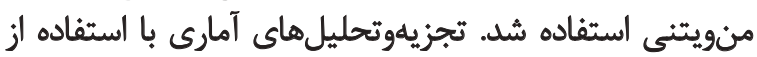
نرم|فزار SPSS نسخه 1 انجام يذئيرفت.

يافتهها

جامعه آمارى يُوهش شامل •ه نفر از دائجويان دانشعاه

1. Pratt
مطالعات انجامشده بيشتر، واكههاى زبان فارسى را در افراد أذراد

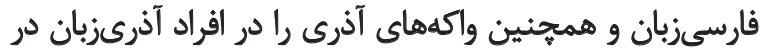

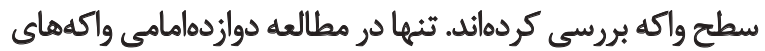

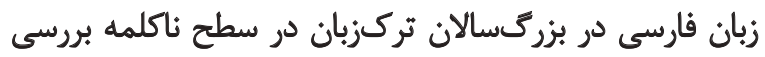

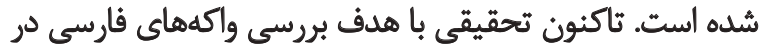

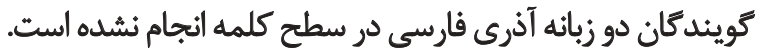

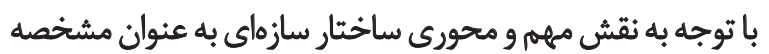

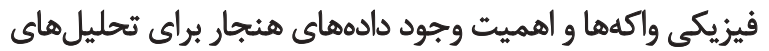

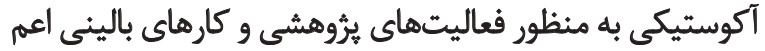

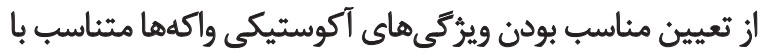

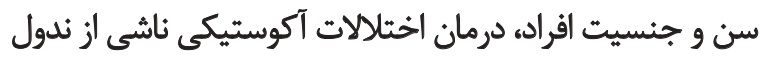

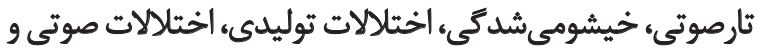

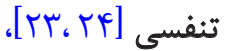

بررسى ساختار سازهاى اهميت زيادى دارد. لذا هدف از اين

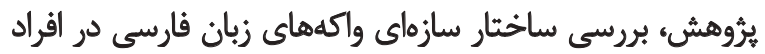

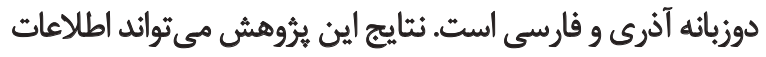

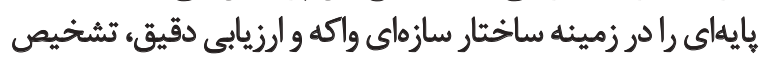

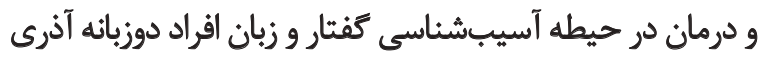

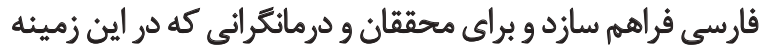
فعاليت مي كثند مفيد و سودمئد باشد.

ووش بروسى

يُروهش حاضر از نوع توصيفى تحليلى بود كه به روشي اذري

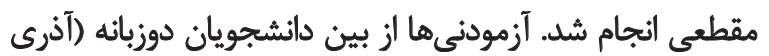

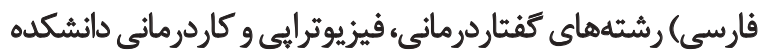

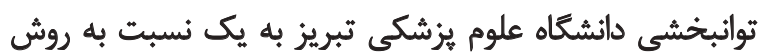

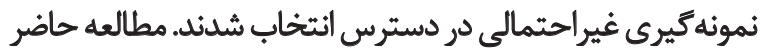

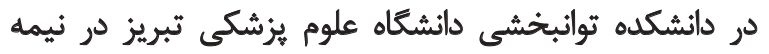

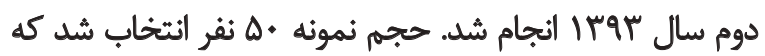

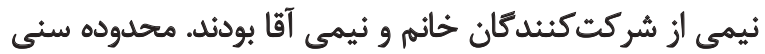

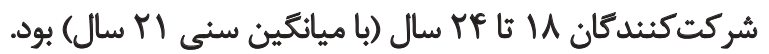

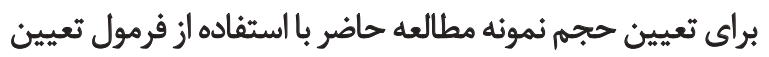

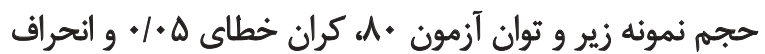

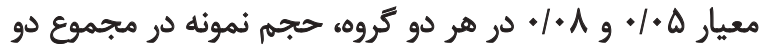

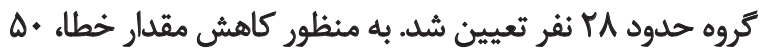

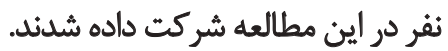
$n=\frac{\left[z_{1-\frac{a}{2}}+z_{1-b}\right]^{2}\left(s_{1}^{2}+s_{1}^{2}\right)}{d^{2}}$

$=\frac{(1.96+0.842)^{2} \times\left((0.05)^{2}+(0.08)^{2}\right)}{0.05^{2}}=27.96$

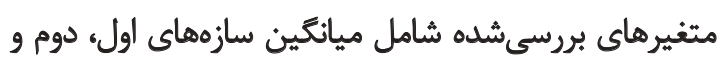

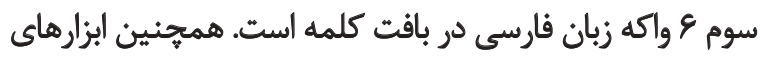




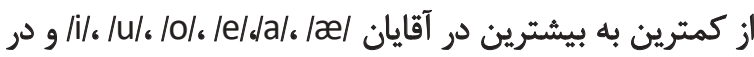

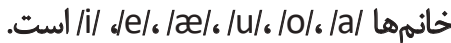

ثt

مطالعه حاضر با هدف بررسى سازههاي اول، دوم و سوم در ع

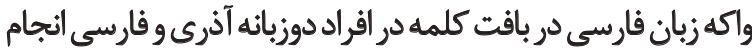

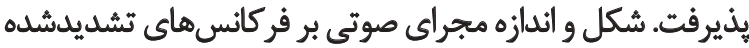

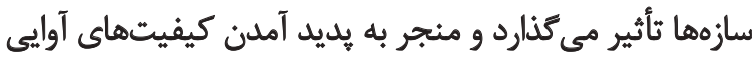

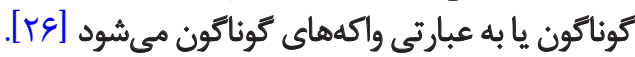
سازه اول نمايانكر ارتفاع زبان در توليد واكههاست. هر خانه

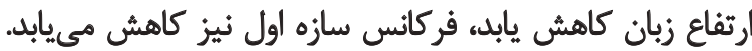

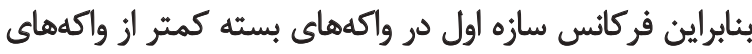

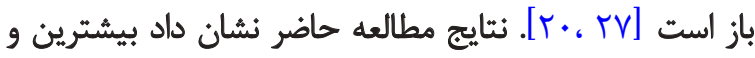

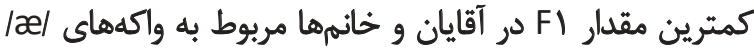

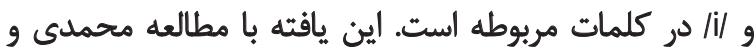

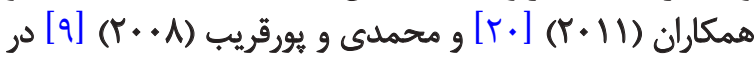

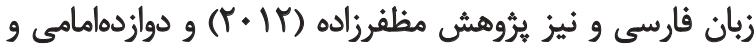

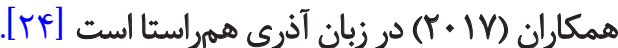

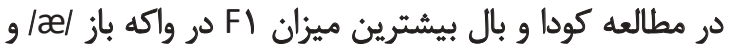

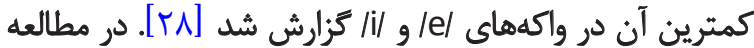
غفاروند كه در زبان آذرى انجام شده بيشترين مقار مقدار

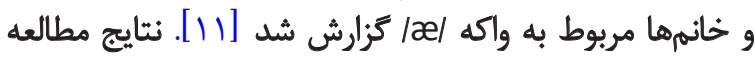

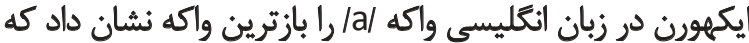

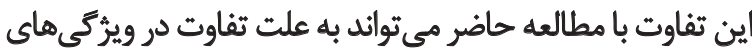

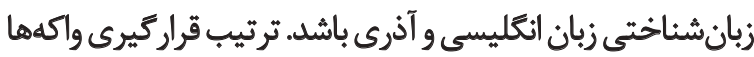

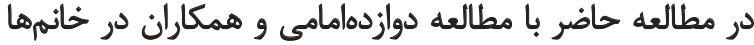

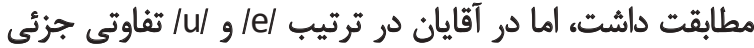

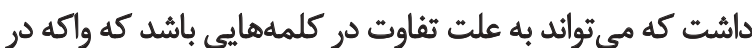
آن برسى شده است.

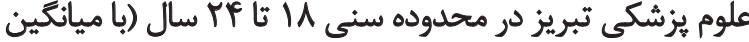

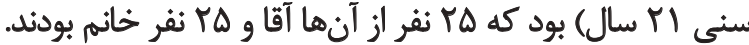

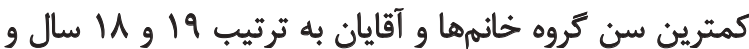

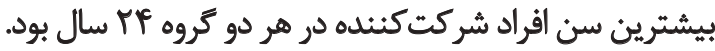
ميانكين FY وF و FY براي هر واكه به تفكيك جنسيت در

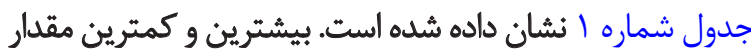

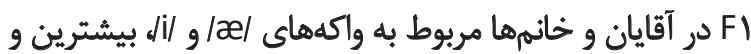

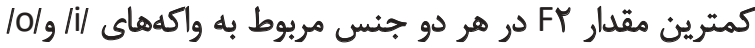

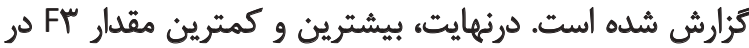

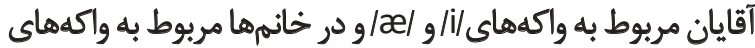

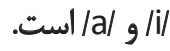

بر اساس دادههاى جدول شماره ا براى مقايسه سازههاى اول

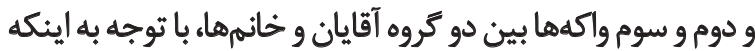

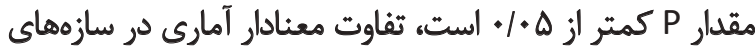

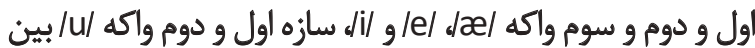

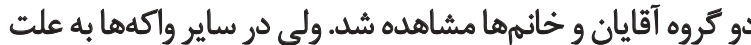

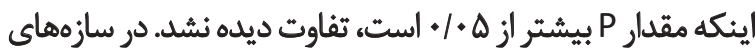

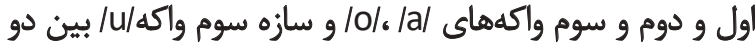

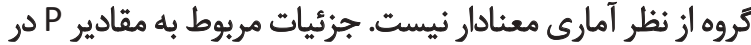
جدول شماره ا نشان داده شده است.

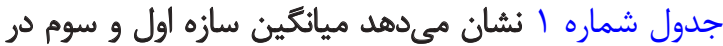

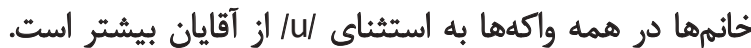

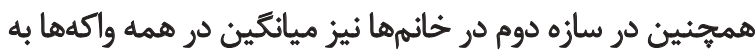

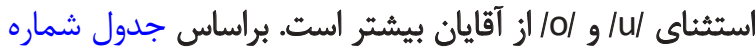

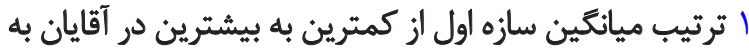

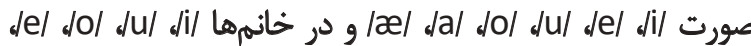

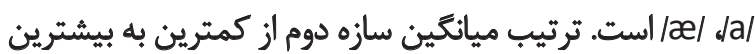

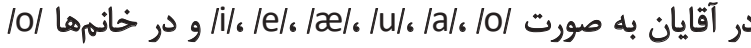
است. همجنين، ترتيب ميانگين سازه سوم

جدولا. ميانكين سازمهاي اول، دوم و سوم واكههاي فارسي در كلمات مدنظر در آقايان و خانهمها

\begin{tabular}{|c|c|c|c|c|c|c|c|c|c|}
\hline \multirow{2}{*}{$\mathbf{P}$} & \multicolumn{2}{|c|}{ سازه سوم } & \multirow{2}{*}{$P$} & \multicolumn{2}{|c|}{ سازه دوم } & \multirow{2}{*}{$P$} & \multicolumn{2}{|c|}{ سازه اول } & \multirow{2}{*}{ واكه در كلمه } \\
\hline & خانمهرا & آقايان & & خاتهمها & آقايان & & خائمهها & آقايان & \\
\hline .1 .01 & TYAS/AT & TAAT/AA & $\%$ & $\mid W /=1$ & IAFNTO & $\%$ & Aft/ar & ENe/D. & / Jær/ در/ / / \\
\hline$\%$ & $r q T \cdot / M r$ & revilir & $\%$ & MTIT/W & $191 F / 14$ & $\%$ & $\Delta r \cdot M$ & $M T / \Delta T$ & / Jer/ در/e/ \\
\hline$\%$ & $\pi T \cdot 1 / 91$ & rqua/Aq & $\%$ & $r \Delta V D / e q$ & MrIVI. & $\% \ldots$ & $M / M T$ & $r+N a V$ & / [ir/ /il/ \\
\hline$. / 5+\varphi^{5}$ & $(r+9 / 4)$ & requ/as & ./FVA & IITNIO & $1114 / 99$ & א ג & $\Delta|N A|$ & $\Delta+r /+r$ & / $/$ or/ $/ 0 /$ \\
\hline$. / r .$. & $(719 / 91$ & TETT/gD &.$/ . \pi r$ & IFMTV & ITPNAT &.$/ M$ & VIV/aY & $\operatorname{seg} / 49$ & / Jar/a/ در/a/ \\
\hline.$/ 14$. & $T Y \Delta \Delta /+$. & rAqT/QS & $.1 . .1$ & $11 F \Delta / A \Delta$ & Iffel.1 & $.1 . .1$ & $P 1 . / N$ & PQV/T. & / /uræ/ در/u/ \\
\hline
\end{tabular}




$$
\text { دارد تا علت آن مشخص شود. }
$$

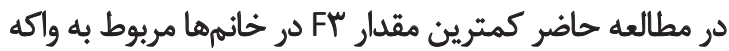

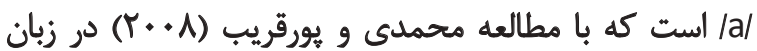

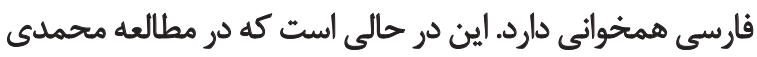

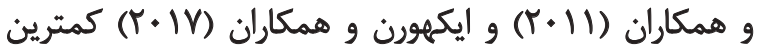

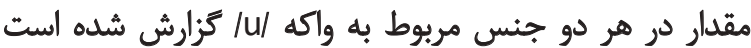

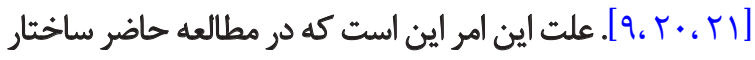

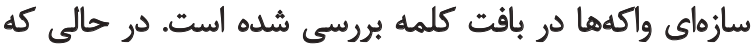

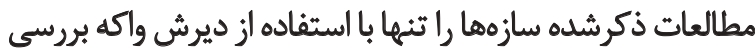

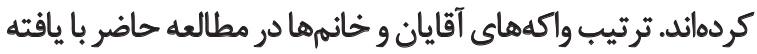

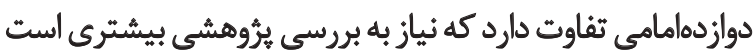

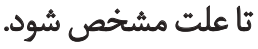

از محدوديتهاى اين يثروهش نبود فضاى آكوستيكى مناسب

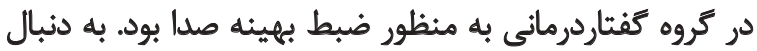

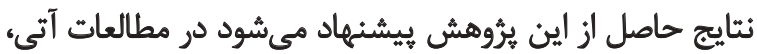

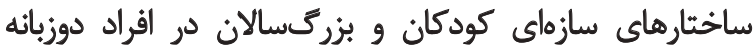

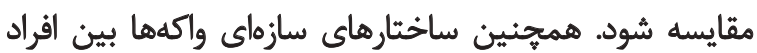

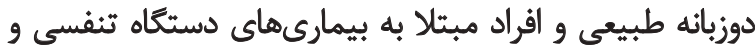
اختلالاتى مانند كمشنوايى و سندرم داون و غيره مقايسه شود.

\section{نتيجليَّى}

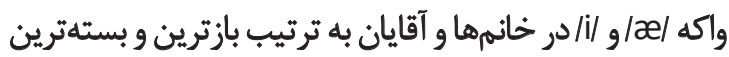

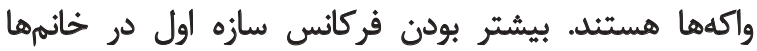

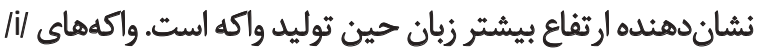

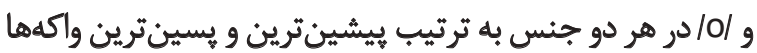

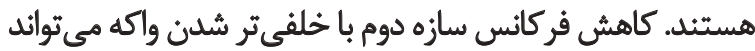

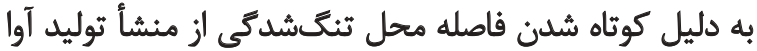

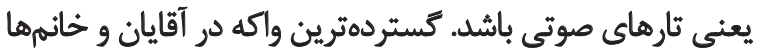

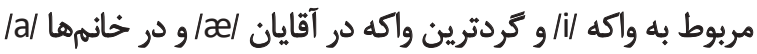

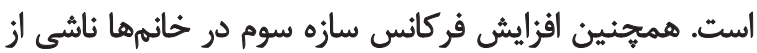
بيشتر بودن دامنه حركتي زبان در خانهماست.

$$
\text { تشيكر و قبدر مانى }
$$

اين مقاله بخشى از يايان نامه مقطع كارشناسى نويسنده مسئول

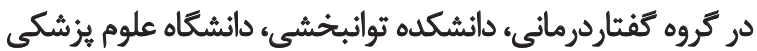

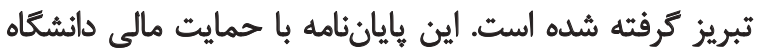

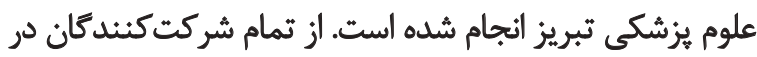

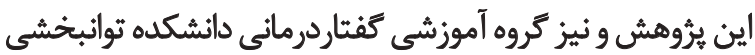

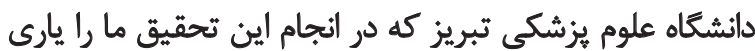

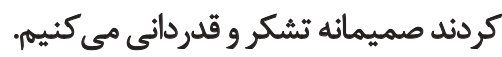

سازه دوم به فاصله تنكَشدكى مسير صوتى تا حنجره مربوط

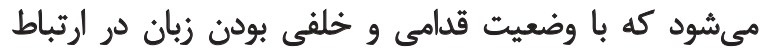

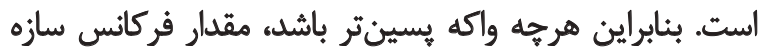

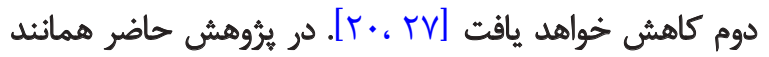

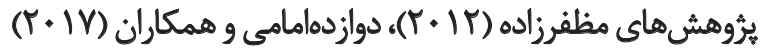

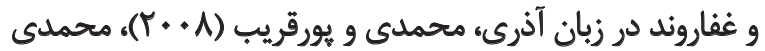

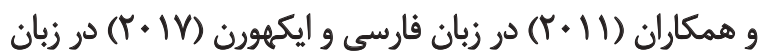

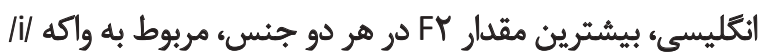

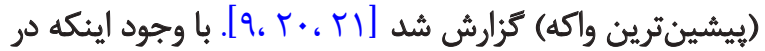

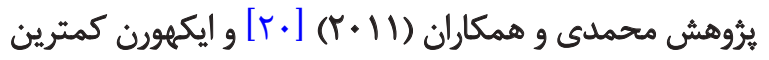

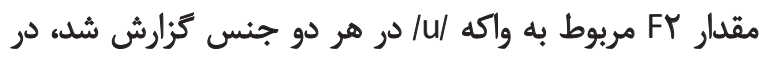

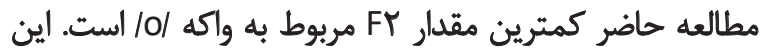

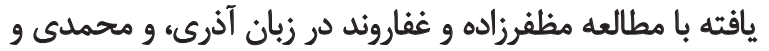

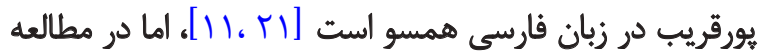

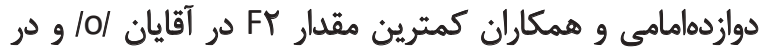

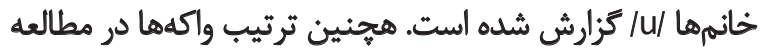

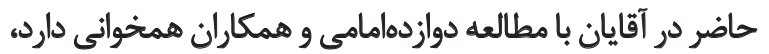

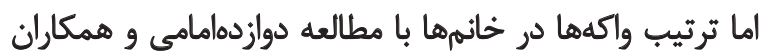

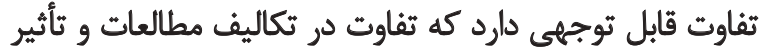
بافت همخوانى مى تواند تاثيركذار باشد.

در اين بررسى مشخص شد در واكههاى بيشين به دليل فاصله

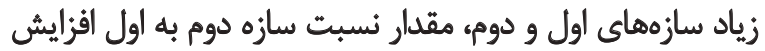

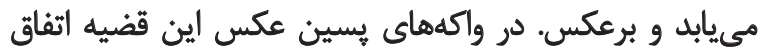

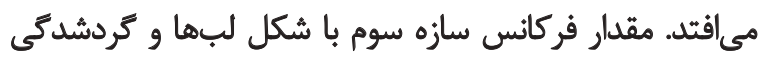

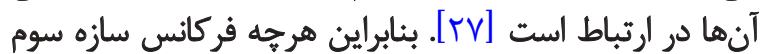

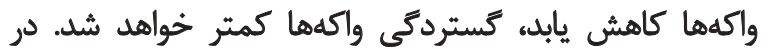

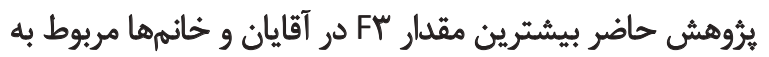

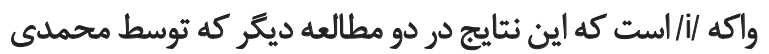

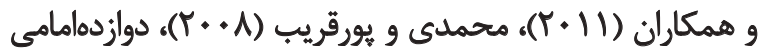

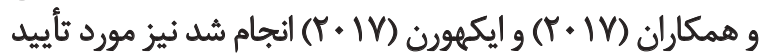

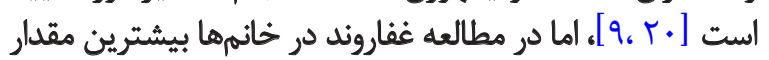

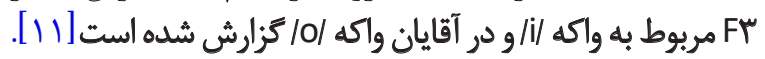

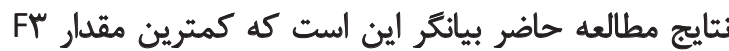

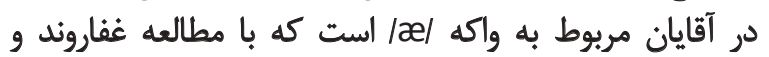

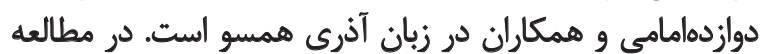

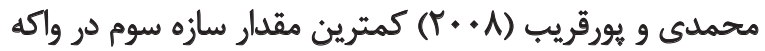

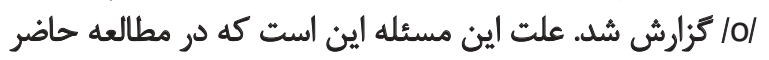

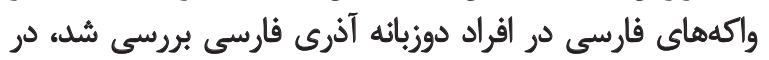

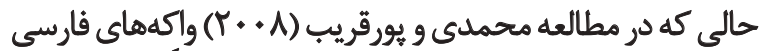

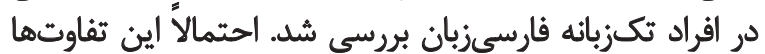

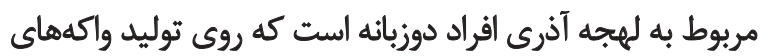

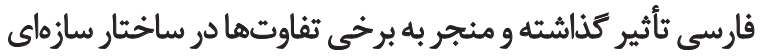

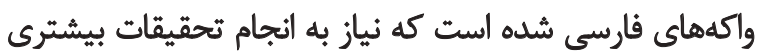




\section{References}

[1] Pisanski K, Rendall D. The prioritization of voice fundamental frequency or formants in listeners' assessments of speaker size, masculinity, and attractiveness. The Journal of the Acoustical Society of America. 2011; 129(4):2201-12. [DOI:10.1121/1.3552866] [PMID]

[2] Aghajanzadeh M, Nikravesh M, Ghorbani H. Survey the frequency of first three formants of Persian vowels in patients with vocal polyps. Journal of Paramedical Sciences \& Rehabilitation. 2015; 4(1):41-8.

[3] Pena Brooks A, Hegde MN. Assessment and treatment of articulation and phonological disorders in children: A dual-level text. Austin, Texas: Pro-Ed Inc; 2007.

[4] Ansarin AA. An acoustic analysis of modern Persian vowels. Paper presented at the $9^{\text {th }}$ Conference Speech and Computer. 20-22 September 2004, Saint-Petersburg, Russia. [PMID]

[5] Nord L, Ericsson G. Acoustic investigation of cleft palate speech before and after speech therapy. Journal of STL-QPSR. 1985; 26(4):15-27.

[6] Johnson K. Acoustic and auditory phonetics. Phonetica. 2004; 61(1):56-8. [DOI:10.1159/000078663]

[7] Ladefoged P, Johnson K. A course in phonetics. Belmont: Thomson Wadsworth; 2006

[8] Yunusova Y, Weismer G, Westbury JR, Lindstrom MJ. Articulatory movements during vowels in speakers with dysarthria and healthy controls. Journal of Speech, Language, and Hearing Research. 2008; 51(3):596-611. [DOI:10.1044/1092-4388(2008/043)]

[9] Mohamadi O, Pourgharib J. Persian vowels formants: An investigation and comparison between Persian children 7-9 years old and Persian adults 18-22 years old. Koomesh. 2008; 9(2):105-9.

[10] Hagino A, Inohara K, Sumita YI, Taniguchi H. Investigation of the factors influencing the outcome of prostheses on speech rehabilitation of mandibulectomy patients. Nihon Hotetsu Shika Gakkai Zasshi. 2008; 52(4):543-9. [DOI:10.2186/jjps.52.543] [PMID]

[11] Mokari PG, Werner S. An acoustic description of spectral and temporal characteristics of Azerbaijani vowels. Poznan Studies in Contemporary Linguistics. 2016; 52(3):503-18. [DOI:10.1515/ psicl-2016-0019]

[12] Shriberg LD, Kent RD. Clinical phonetics. London: Pearson; 2003.

[13] Baken RJ, Orlikoff RF. Clinical measurement of speech and voice. Boston: Cengage Learning; 2000.

[14] Natour YS, Marie BS, Saleem MA, Tadros YK. Formant frequency characteristics in normal Arabic-speaking Jordanians. Journal of Voice. 2011; 25(2):e75-e84. [DOI:10.1016/j. jvoice.2010.10.018] [PMID]

[15] Jalilvand-Karimi L, Motlaghzadeh L, Mohammadkhani G, Baghban AA. The comparison of auditory capacity between bilinguals and monolinguals by consonant-vowel dichotic test. Rehabilitation Medicine. 2014; 2(3):19-30. [DOI:10.22037/r.m.v2i3.5693]
[16] Hakimi A. Comparative phonetic study of frequently used words in Iranian Farsi versus Tajik Farsi. Journal of American Science. 2012; 8(4):6-16.

[17] Peterson GE, Barney HL. Control methods used in a study of the vowels. The Journal of the Acoustical Society of America. 1952; 24(2):175-84. [DOI:10.1121/1.1906875]

[18] Eichhorn JT, Kent RD, Austin D, Vorperian HK. Effects of aging on vocal fundamental frequency and vowel formants in men and women. Journal of Voice. 2017; pii: S0892-1997(17)30161-3. [DOI:10.1016/j.jvoice.2017.08.003] [PMID]

[19] Mohammadi R, Mohammadi B. [Studying the Persian vowel formants in students of Rehabilitation School, Iran University of Medical Sciences (Persian)] [PhD dissertation]. Tehran: Iran University of Medical Sciences; 2003.

[20] Mohammadi H, Mohammadi R, Torabinezhad F, Rezaei M. [Formant structure and vowel space in Persian vowels (Persian)]. Bimonthly Audiology-Tehran University of Medical Sciences. 2011; 20(2):79-85.

[21] Peivasti SM. An acoustic analysis of Azerbaijani vowels in Tabrizi dialect. Journal of Basic and Applied Scientific Research. 2012; 2:7181-4.

[22] Davazdah Emami A, Ghelichi L, Jenabi M, Amiri SY, Modaresi Y, Kamali M. [A comparative study of Persian vowel quality in the speech of 18-28 year old Turkish speakers (Persian)]. Middle Eastern Journal of Disability Studies. 2017; 7:27.

[23] Dehqan A, Ansari H, Bakhtiar M. Objective voice analysis of Iranian speakers with normal voices. Journal of Voice. 2010; 24(2):161-7. [DOI:10.1016/j.jvoice.2008.07.005] [PMID]

[24] Peivasti SM. An acoustic analysis of Azerbaijani vowels in Tabrizi dialect. Journal of Basic and Applied Scientific Research. 2012; 2(7):7181-4.

[25] Casper JK, Leonard R. Understanding voice problems: A physiological perspective for diagnosis and treatment. Philadelphia: Lippincott Williams \& Wilkins; 2006.

[26] Salehi F, Bahrami A, Pourgharib J, Torabinezhad F, Kamali M. [The Persian vowel formants in normal, moderate and severe hearing impaired students age 7-9 years in Isfahan (Persian)]. Audiology. 2009; 17(2):42-52.

[27] Samare Y. [Phoneticism of Persian language (sounds and soundtrack) (Persian)]. Tehran: Markaz-e Nashr-e Daneshgahi; 1999.

[28] Ball MJ, Gröne B. Imaging techniques. In: Ball MJ, Code C, editors. Instrumental Clinical Phonetics. London: Whurr; 1997. [DOI:10.1002/9780470699119] 
